\title{
Spectrophotometric multicomponent analysis of irbesartan, hydrochlorothiazide and ramipril in pharmaceutical formulations by chemometric techniques
}

\begin{abstract}
Partial Least Squares (PLS) and Principal Component Regression (PCR) methods were proposed for the spectrophotometric analysis of a ternary mixture consisting of Irbesartan, Hydrochlorothiazide and Ramipril without prior separation. In these chemometric techniques the measurements of absorbance values were realized in the spectral range from $200-350 \mathrm{~nm}$ in the intervals of $\mathrm{Dl}=1 \mathrm{~nm}$ at the 151 wavelengths in the zero order, first and second order spectra of the different ternary mixtures of these ingredients in $0.1 \mathrm{M} \mathrm{NaOH}$. The prepared calibrations of both techniques using the absorbance data and concentration matrix data sets were used to predict the concentrations of the unknown concentrations of Irbesartan, Hydrochlorothiazide and Ramipril in their ternary mixture. The techniques were successfully applied to pharmaceutical formulations marked in India.
\end{abstract}

Keywords: irbesartan, hydrochlorothiazide, ramipril, chemometrics, simultaneous estimation, calibrations, absorbance, chemometric, spectrophotometric
Volume 2 Issue 3 - 2016

\author{
Lakshmi Sivasubramanian,' KS Lakshmi ${ }^{2}$ \\ 'Department of Pharmaceutical Analysis, JNTUA University, \\ India \\ ${ }^{2}$ Department of Pharmaceutical Analysis, SRM University, India
}

Correspondence: Lakshmi Sivasubramanian, Department of Pharmaceutical Analysis, Sri Venkateswara College of Pharmacy, JNTUA University, RVS Nagar, Chittoor - 517127,AP, India, Email lakshmiss@hotmail.com

Received: April 23, 2016 | Published: May 09, 2016
Abbreviations: PLS, partial least squares; PCR, principal component regression; IRB, irbesartan; $\mathrm{HCZ}$, hydrochlorothiazide; RAM, ramipril; HPLC, high performance liquid chromatography; PLS, partial least squares regression; SEP, standard error prediction

\section{Introduction}

For the determination of Irbesartan (IRB), Hydrochlorothiazide (HCZ) and Ramipril (RAM), different analytical methods such as spectrophotometry, ${ }^{1-6}$ spectrofluorimetry, ${ }^{7,8}$ chemometrics,,${ }^{9-14}$ high Performance Liquid Chromatography (HPLC), ${ }^{15-35}$ LC-MS, ${ }^{36-41}$ capillary zone electrophoresis ${ }^{42}$ and High performance thin layer chromatography (HPTLC) ${ }^{43-47}$ were reported individually or in combination with other drugs. But no method was reported so far for the simultaneous analysis of these drugs in ternary mixture. In comparison with conventional univariate methods, Multivariate spectrophotometric methods provide several advantages as it includes multiple variables for data analysis. These methods overcome the limitations of conventional techniques thereby applied to the simultaneous quantification of multiple analytes in mixtures.

For quantitative analysis of many pharmaceutical formulations, multivariate calibration methods like Partial Least Squares Regression (PLS) and Principal Component Regression (PCR) are predominantly used. These methods are based on the association between matricial algorithms and calibration data and the theoretical base of these methods are not fully explored by authors.

Due to the spectral overlapping simultaneous estimation of three components becomes difficult by conventional methods. Derivative spectrophotometry offers an effective quantification of multicomponent samples from their mixtures, as they resolve the common overlapping seen in the fundamental spectra. Hence derivative spectrophotometry offers greater sensitivity than the normal spectrophotometry in the simultaneous determination of two components without prior separation. An attempt has been made to overcome such spectral overlapping felt by three components in a ternary mixture by the combined use of derivative spectrophotometry and chemometric techniques.

Two multivariate procedures such as PCR and PLS algorithms are reported for the ternary mixture of drugs of interest. An effort was also made to compare the regression methods for fundamental spectra with that of first and second derivative spectra. From a calibration set comprising of 25 reference mixtures of selected analytes, the calibration models were built using a novel experimental design. In order to verify the prediction ability of the defined models, and external validation test was performed by using prediction set comprising of 10 synthetic mixtures of three analytes. The proposed methods were finally applied to the quantitative analysis of commercial samples containing one to three drugs to confirm their effectiveness in the routine quality control analysis of the real samples.

\section{Materials and methods}

\section{Apparatus}

A Perkin Elmer (Lamda 25) spectrophotometer controlled by UV winlab software and equipped with $1 \mathrm{~cm}$ pathlength quartz cell was used for the spectral data acquisition. The chemometric procedure was carried out using MATLAB software version 7.5 (The Mathworks) and PLS toolbox version 5.0 (Eigen Vector Technologies).

\section{Chemicals}

IRB, HCZ and RAM reference standards were kindly supplied by Madras Pharmaceuticals, Chennai, India. The tablets IROVEL H, Sun Pharmaceuticals, XARB-H, Hetero Labs (both containing 150 $\mathrm{mg}$ of IRB and $12.5 \mathrm{mg}$ of HCZ) and Cardace\#2.5, Aventi Pharma (containing $2.5 \mathrm{mg}$ of RAM and $12.5 \mathrm{mg}$ of $\mathrm{HCZ}$ ) were procured from local pharmacies. All other chemicals were of analytical reagent grade and procured from SD Fine chemicals, Mumbai, India. 


\section{Standard solutions}

Standard stock solutions $(1000 \mu \mathrm{g} / \mathrm{ml})$ of IRB, HCZ and RAM were prepared separately in the diluent $0.1 \mathrm{M} \mathrm{NaOH}$ and water in the ratio 20:80. These solutions were taken and then diluted to $10 \mathrm{ml}$ with water to give a final analyte concentration desired.

\section{Sample preparation}

Ten tablets were weighed and finely powdered in a mortar. A quantity of the powder equivalent to one tablet was accurately weighed and transferred into a $100 \mathrm{ml}$ volumetric flask including the diluent. The flask was sonicated for $15 \mathrm{mins}$ and diluted to the mark with diluents. An aliquot of the solution was centrifuged at $5000 \mathrm{rpm}$ for 10mins. Appropriate amount of clear supernatant was transferred into a $10 \mathrm{ml}$ flask and diluted with water. Then the absorbance values were measured.

\section{Methods}

\section{Multivariate calibration methods}

In multivariate calibration methods, a calibration model is built using the absorbance values and a concentration data set. These methods are designed based on the relationship between matrices of chemical data. Initially a calibration model is built using a chemical data set i.e. absorbance values and a concentration data set. The constructed calibration model is then used to estimate the concentration of the unknown samples in the prediction set. In the PLS and PCR methods, the original variables are transformed in to smaller number of orthogonal variables called factors or principal components which are in turn linear with the original variables.

From the prepared reference samples, a relationship between spectral and concentration data, representing the variables of the system can be built using the multivariate calibration techniques. Based on the regression method, the PCs and scores can be built specific to the new matrix. The PCs corresponds to the absorptivity values of the samples at various concentration and wavelength values, whereas the scores corresponds the numerical coefficients. This allows to build the mathematical model representing the reference spectra and able to predict the component concentrations of new samples.

\section{Determination of PC number}

The prediction power of the selected methods can be improved by determining the optimal number of factors. This can be achieved by employing a full cross validation also known as leave-one-out method. This method employs leaving one sample at a time from the calibration step and performs the calibration with all other samples. The concentration of the sample removed is then predicted with the obtained model. This step is in turn repeated for each sample considered. The procedure can be repeated after fixing a different number of factors. The standard error prediction (SEP) was chosen as an optimizing criterion to select the optimal number of factors. SEP represents an estimate of the error involved in the assay of external samples by using the model. Its value depends on the number of factors used for that calibration. The number of factors giving the minimal SEP was selected as the optimal number of factors.

$$
R M S E P=\sqrt{\frac{\sum_{i=1}^{n}\left(\frac{\hat{c}}{c_{i}^{2}}\right)}{n}}
$$

Where $\hat{c}$ denotes the added drug concentration, $C i$ is the predicted drug concentration and $n$ represents the total number of synthetic mixtures.
Table I Concentrations of IRB, HCZ and RAM used as Calibration and Prediction sets

\begin{tabular}{|c|c|c|c|c|c|}
\hline \multicolumn{3}{|c|}{ Calibration set $(\mu \mathrm{g} / \mathrm{ml})$} & \multicolumn{3}{|c|}{ Prediction set $(\mu \mathrm{g} / \mathrm{ml})$} \\
\hline IRB & HCZ & RAM & IRB & HCZ & RAM \\
\hline 0.5 & 0.5 & 5 & 2 & 0.5 & 1.5 \\
\hline I & 0.5 & 4 & 2 & 1 & 2.5 \\
\hline 1.5 & 0.5 & 3 & 1 & 1.5 & 2 \\
\hline 2 & 0.5 & 2 & 1.5 & 2 & 2.5 \\
\hline 0.5 & 1 & 5 & 0 & 2.5 & 1.5 \\
\hline I & I & 4 & 0.5 & 3 & 4 \\
\hline 1.5 & 1 & 3 & 1.5 & 3 & 4 \\
\hline 2 & 1 & 2 & 0 & 0 & 3 \\
\hline 0.5 & 0.5 & 5 & 0 & 1 & 6 \\
\hline I & I.5 & 4 & 1 & 2 & 1.5 \\
\hline 1.5 & 1.5 & 3 & - & - & - \\
\hline 2 & 1.5 & 2 & - & - & - \\
\hline 0 & 2 & 0 & - & - & - \\
\hline 0.5 & 2 & 5 & - & - & - \\
\hline I & 2 & 4 & - & - & - \\
\hline 1.5 & 2 & 3 & - & - & - \\
\hline 0.5 & 2.5 & 5 & - & - & - \\
\hline I & 2.5 & 4 & - & - & - \\
\hline 1.5 & 2.5 & 3 & - & - & - \\
\hline 2 & 2.5 & 2 & - & - & - \\
\hline 0.5 & 3 & I & - & - & - \\
\hline I & 3 & 1.5 & - & - & - \\
\hline 0 & 0 & 6 & - & - & - \\
\hline 3 & 0 & 0 & - & - & - \\
\hline 1.5 & 3 & 2 & - & - & - \\
\hline
\end{tabular}

\section{Results and discussion}

\section{Data Processing and model building}

The UV absorption spectra of standard IRB, HCZ and RAM in $0.1 \mathrm{M} \mathrm{NaOH}$ is recorded in the range of $200-350 \mathrm{~nm}$ in ordinary mode (Figure 1a), First derivative mode (Figure 1b) and second derivative mode (Figure 1c). Specific wavelengths for these components could not be signaled out from all the recorded spectra. Hence multivariate calibration methods appeared to be ideal to overcome the drawbacks, as the entire information from the full spectra is being utilized by these techniques.

A concentration of 25 mixtures of three compounds in the range of $0.5-3 \mu \mathrm{g} / \mathrm{ml}$ of IRB and $\mathrm{HCZ}$ and $1-6 \mu \mathrm{g} / \mathrm{ml}$ of RAM were prepared in $0.1 \mathrm{M} \mathrm{NaOH}$ (Table 1). The fundamental, first derivative and second derivative spectra of the proposed concentration set were recorded in the $200-350 \mathrm{~nm}$ wavelength range. The absorption values of spectra of the concentration set were measured at the 151 wavelength points with $\mathrm{Dl}=1 \mathrm{~nm}$ in the spectral region of $200-350 \mathrm{~nm}$. The concentration set and absorption data were considered as Y-block $(25 \times 3)$ and X-block $(25 \times 151)$ for the construction of PLS calibration using cross-validation procedure to reach the best calibration model. The calculations were done with PLS Toolbox 5.0 and the optimal factors were selected.

The PCR and PLS models obtained by using this training set were validated by full cross-validation and the SEP values were calculated each time that a new factor was added to the models. Table $2 \&$ Figure 2 shows the selected numbers of factors and the corresponding SEP values. The square of the correlation coefficient $(R 2)$, which indicates the fraction of the total variance explained by the models, is also reported. The prediction values for IRB, HCZ and RAM in both models were found satisfactory (Figure 3 ). 


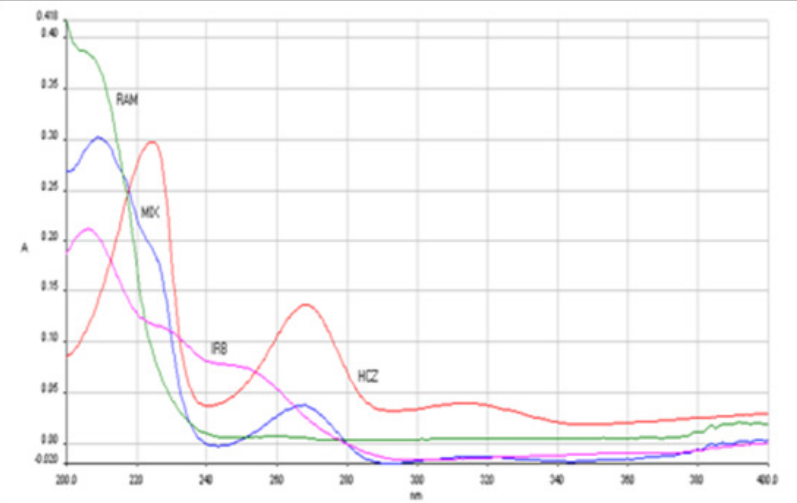

Figure I a FundamentalAbsorption spectra of Irbesartan, Hydrochlorothiazide, Ramipril and mixture.

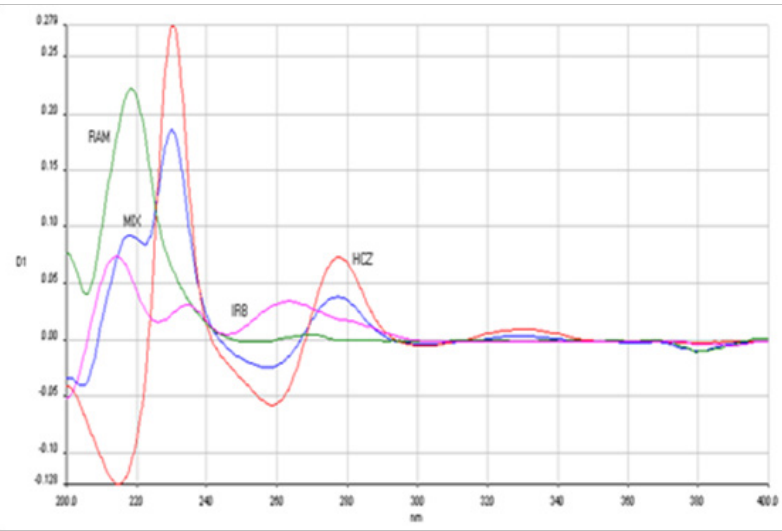

Figure Ib First derivative spectra of Irbesartan, Hydrochlorothiazide, Ramipril and mixture.

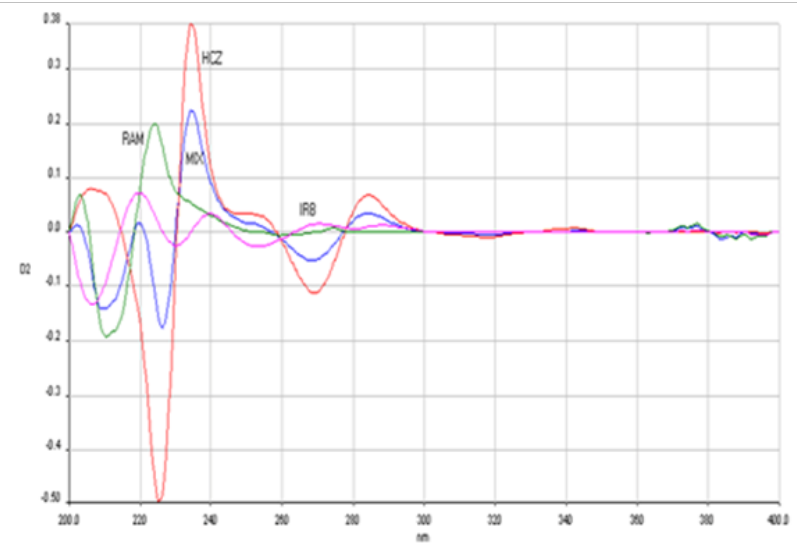

Figure Ic Second derivative spectra of Irbesartan, Hydrochlorothiazide, Ramipril and mixture.

\section{Application of PLS and PCR models to the Prediction set}

In order to perform an external validation for the proposed PLS and PCR models, a set of 10 synthetic samples containing ternary and binary component mixtures of these selected drugs were prepared. The mixtures were prepared in the same concentration range as in the calibration set (Table 1). Such constructed prediction set was used to test the prediction ability of the models. The application of proposed PLS and PCR models to the prediction set gave the following results and are indicated in Table 3 \& Figure 4.

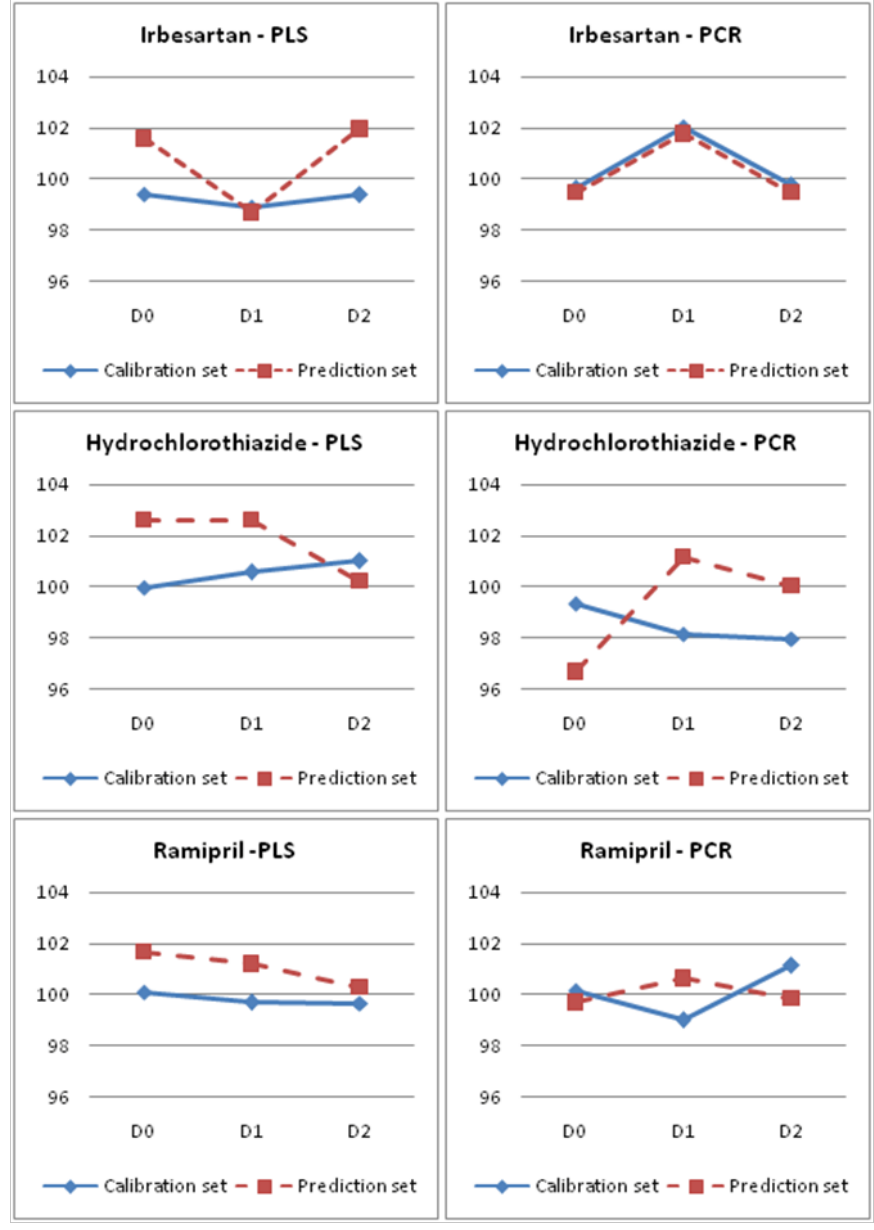

Figure 2 Comparison of Mean Recovery of PLS and PCR models for Fundamental (D0), First Derivative (DI) and Second Derivative (D2) Spectra.

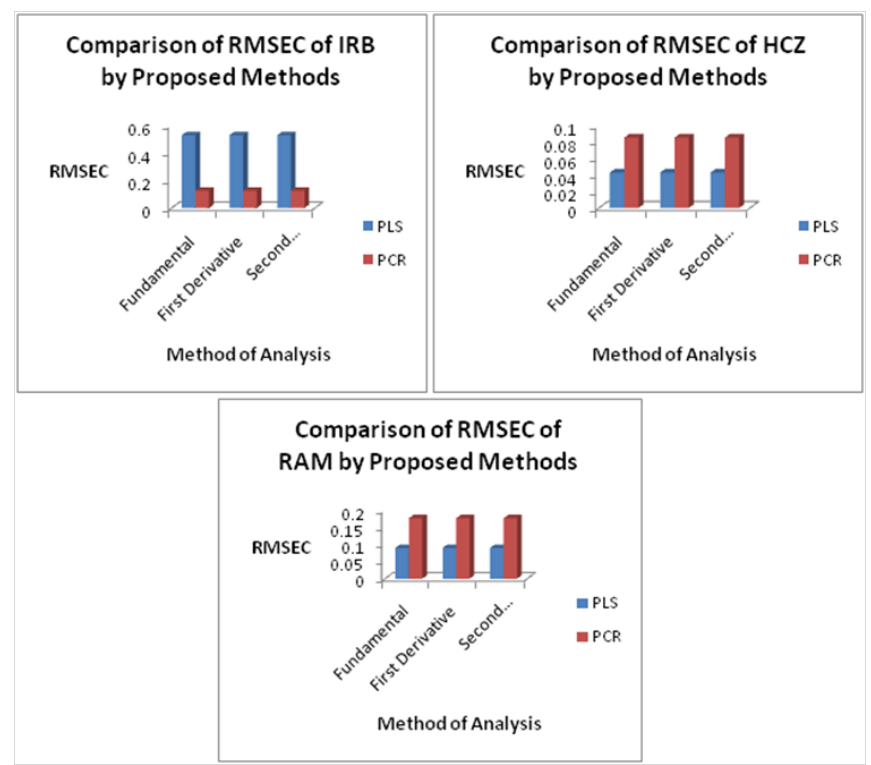

Figure 3 Comparison of Root Mean Square Error of Calibration (RMSEC) of PLS and PCR models for Fundamental (D0), First Derivative (DI) and Second Derivative (D2) Spectra. 


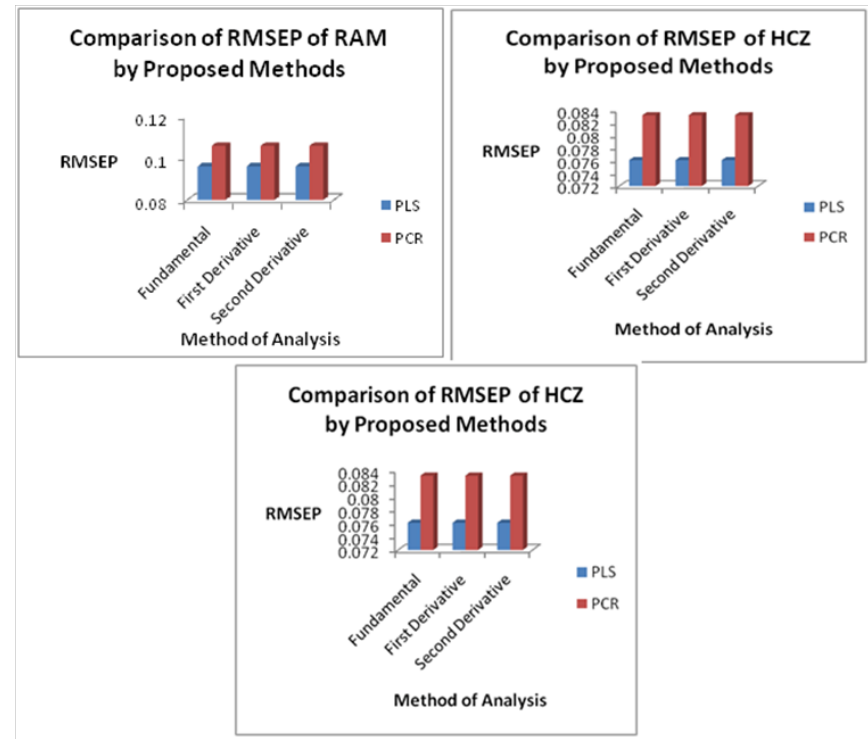

Figure 4 Comparison of Root Mean Square Error of Prediction (RMSEP) of PLS and PCR models for Fundamental (D0), First Derivative (DI) and Second Derivative (D2) Spectra.

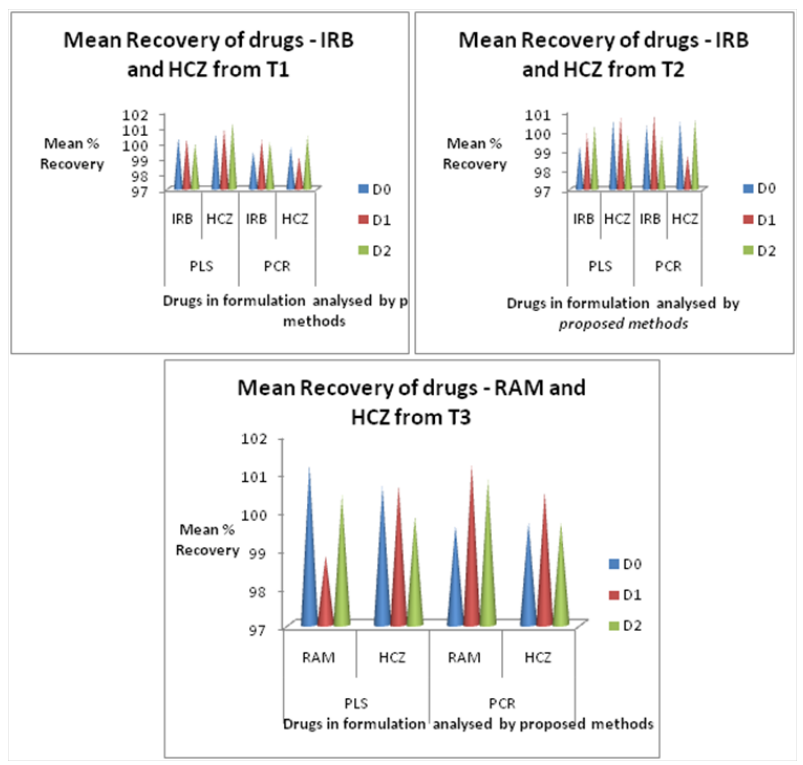

Figure 5 Comparison of \% Recovery of drugs from formulations by PLS and PCR models for Fundamental (D0), First Derivative (DI) and Second Derivative (D2) Spectra.

Table 2 Statistical Parameters calculated from application of PLS and PCR methods to calibration samples

\begin{tabular}{|c|c|c|c|c|c|c|c|}
\hline \multirow{2}{*}{ Drug } & \multirow{2}{*}{ Parameter } & \multicolumn{3}{|l|}{ PLS } & \multicolumn{3}{|l|}{ PCR } \\
\hline & & $\mathbf{D}^{0}$ & $\mathbf{D}^{\prime}$ & $\mathbf{D}^{2}$ & $\mathbf{D}^{0}$ & $\mathbf{D}^{\prime}$ & $\mathbf{D}^{2}$ \\
\hline \multirow{4}{*}{ IRB } & Mean & 99.436 & 98.919 & 99.396 & 99.634 & 99.634 & 99.634 \\
\hline & RMSEC & 0.5298 & 0.5298 & 0.5298 & 0.1262 & 0.1262 & 0.1262 \\
\hline & $\mathrm{R}^{2}$ & 0.9943 & 0.9943 & 0.9943 & 0.9685 & 0.9685 & 0.9685 \\
\hline & Mean & 99.979 & 100.576 & 101.018 & 99.34 & 99.34 & 99.34 \\
\hline \multirow[t]{3}{*}{$\mathrm{HCZ}$} & RMSEC & 0.0431 & 0.0431 & 0.0431 & 0.0857 & 0.0857 & 0.0857 \\
\hline & $\mathrm{R}^{2}$ & 0.9979 & 0.9979 & 0.9979 & 0.9919 & 0.9919 & 0.9919 \\
\hline & Mean & 100.111 & 99.695 & 99.689 & 100.165 & 100.165 & 100.165 \\
\hline \multirow[t]{2}{*}{ RAM } & RMSEC & 0.0892 & 0.0892 & 0.0892 & 0.1761 & 0.1761 & 0.1761 \\
\hline & $\mathrm{R}^{2}$ & 0.9968 & 0.9968 & 0.9968 & 0.9877 & 0.9877 & 0.9877 \\
\hline
\end{tabular}

Mean, Mean of 25 samples of calibration set; RMSEC, root mean square error of calibration set;

$\mathrm{R}^{2}$, correlation coefficient obtained by plotting amount present against amount estimated.

Table 3 Accuracy (\% recovery) and Precision (SD) results from application of optimized PLS and PCR models on the prediction set

\begin{tabular}{|c|c|c|c|c|c|c|c|}
\hline \multirow{2}{*}{ Drug } & \multirow{2}{*}{ Parameter } & \multicolumn{3}{|l|}{ PLS } & \multicolumn{3}{|l|}{ PCR } \\
\hline & & $\mathbf{D}^{0}$ & $\mathbf{D}^{\prime}$ & $D^{2}$ & $\mathbf{D}^{0}$ & $D^{\prime}$ & $\mathbf{D}^{2}$ \\
\hline & Mean \% Recovery & 101.5939 & 101.5939 & 101.5939 & 99.0195 & 99.0195 & 99.0195 \\
\hline \multirow[t]{3}{*}{ IRB } & RMSEP & 0.1481 & 0.1481 & 0.1481 & 0.0808 & 0.0808 & 0.0808 \\
\hline & SD & 5.8949 & 5.8949 & 5.8949 & 4.4397 & 4.4397 & 4.4397 \\
\hline & Mean \% Recovery & 102.6194 & 102.6194 & 102.6194 & 96.6958 & 96.6958 & 96.6958 \\
\hline \multirow[t]{3}{*}{$\mathrm{HCZ}$} & RMSEP & 0.0761 & 0.0761 & 0.0761 & 0.0832 & 0.0832 & 0.0832 \\
\hline & SD & 4.8994 & 4.8994 & 4.8994 & 6.3584 & 6.3584 & 6.3584 \\
\hline & Mean \% Recovery & 101.6947 & 101.6947 & 101.6947 & 99.6934 & 99.6934 & 99.6934 \\
\hline \multirow[t]{2}{*}{ RAM } & RMSEP & 0.0961 & 0.0961 & 0.0961 & 0.1059 & 0.1059 & 0.1059 \\
\hline & SD & 2.647 & 2.647 & 2.647 & 3.7579 & 3.7579 & 3.7579 \\
\hline
\end{tabular}

Mean \% Recovery, mean of 10 samples of prediction set; RMSEP, root mean square error of prediction set; SD, standard deviation

The results of PLS model includes mean \% recovery ranging from 98.919 to 101.018 for all the three drugs in the calibration set and from 98.704 to 102.619 in the prediction set. The SD values were found to increase with increasing spectral mode in the calibration set but seem to decrease with increasing spectral mode in the prediction set. $\mathrm{R}^{2}$ value ranges from $0.9932-0.9979$ in the calibration set and from $0.9635-0.9988$ in the prediction set. RMSEC value ranges from 0.0431-0.1302 for all three drugs in the calibration set. RMSEP value ranges from $0.0469-0.1481$ for all three drugs in the prediction set.
The results of PCR model includes mean \% recovery ranging from 98.919 to 101.018 for all the three drugs in the calibration set and from 98.704 to 102.619 in the prediction set. The SD values were found to increase with increasing spectral mode in the calibration set but seem to decrease with increasing spectral mode in the prediction set. $\mathrm{R}^{2}$ value ranges from 0.9932-0.9979 in the calibration set and from $0.9635-0.9988$ in the prediction set. RMSEC value ranges from 0.0431-0.1302 for all three drugs in the calibration set. RMSEP value ranges from $0.0469-0.1481$ for all three drugs in the prediction set. 
Table 4 Assay Results of optimized PLS and PCR models on Pharmaceutical preparations

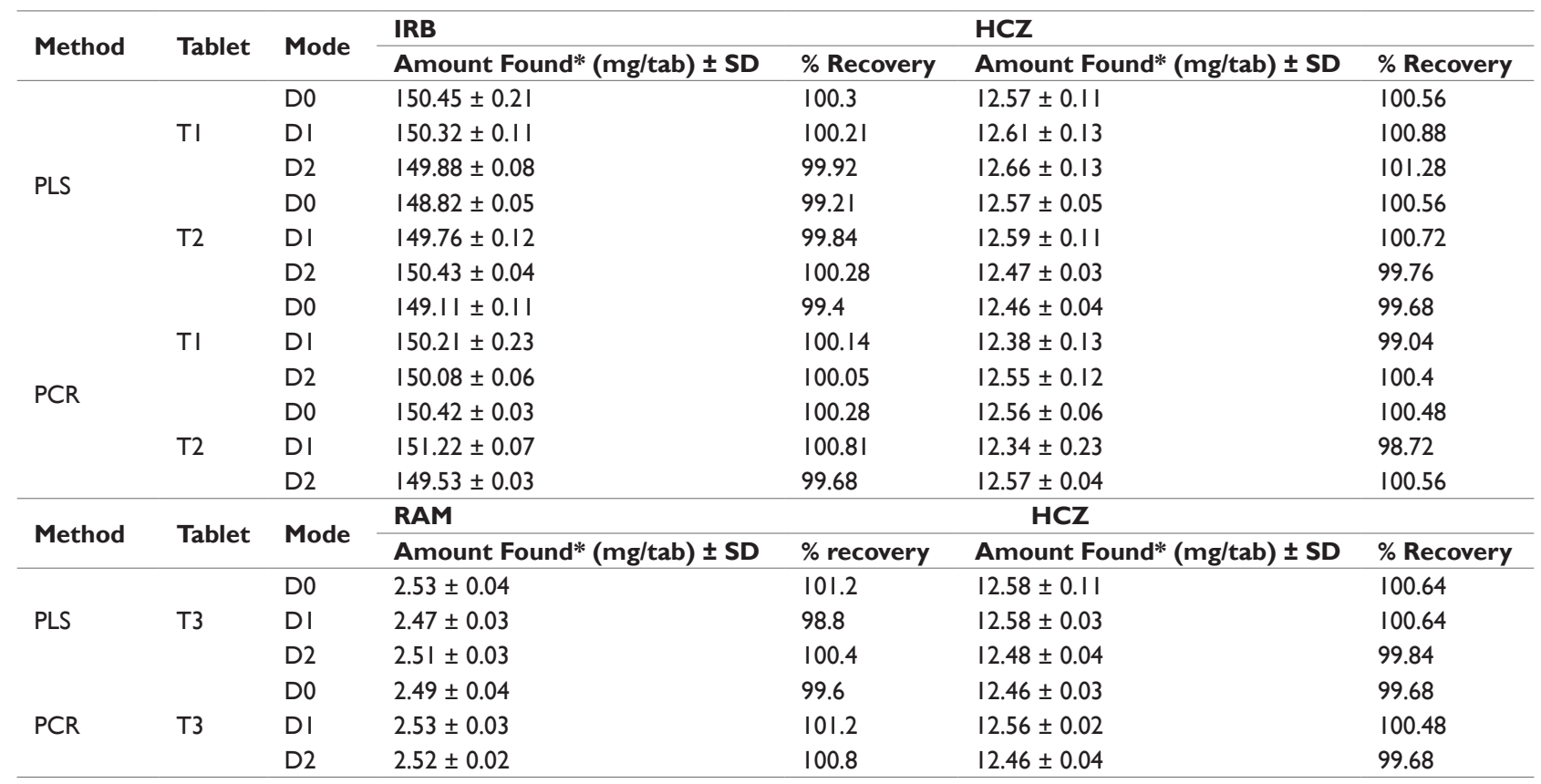

*Average of six estimations

TI - IROVEL H - Sun Pharmaceuticals, J\&K, Irbesartan- I50 mg and Hydrochlorothiazide- 12.5 mg

T2 - XARB H - Hetero Labs, HP, Irbesartan- 150 mg and Hydrochlorothiazide- 12.5 mg

T3 - Cardace \# 2.5 - Aventis Pharma, Goa, Ramipril-2.5 mg and Hydrochlorothiazide- 12.5 mg

Table Abbreviations: PLS, partial least square; PCR, principal component regression; IRB, irbesartan; HCZ, hydrochlorothiazide; RAM, ramipril; $D^{0}$, fundamental/ zero order; $D^{\prime}$, first order; $D^{2}$, second order; RMSEC, root mean square error of calibration; RMSEP, root mean square error of prediction; SD, standard deviation; $\mathrm{R}^{2}$, correlation coefficient

\section{Analysis of commercial formulations}

The proposed PLS and PCR models were applied to the assay of binary pharmaceutical formulations. The assay results are summarized in Table $4 \&$ Figure 5. A good coincidence was observed between experimental results and label claim of the pharmaceutical formulations. Application of models from fundamental to second derivative spectra gave recovery between 98.72 to $101.20 \%$ and SD of not more than 1.0. No interference from the excipients was observed.

\section{Conclusion}

The spectrophotometric data of complex mixtures results in severe spectral overlapping thereby imposing difficulty in simultaneous analyses. Multivariate methods such as PCR and PLS overcomes the difficulty and proves to be valid analytical tools for quantitative estimation of analytes from complex mixtures. The prediction ability of the multivariate methods can be further magnified by acquisition of derivative spectra. The application of PLS and PCR methods on zero to second order derivative spectra of ternary mixtures of IRB, $\mathrm{HCZ}$ and RAM has been accomplished. Very satisfactory results were obtained when the optimized models were applied to the synthetic mixtures and commercial formulations. According to these studies, multivariate calibration methods (PLS and PCR) coupled with derivative spectral data can be recommended as a very suitable choice to resolve severe overlapped absorption spectra of drug mixtures. This approach is simple in application, inexpensive, requires an easy treatment of the samples and provides reliable analytical results.

\section{Acknowledgments}

The authors are thankful to the management of SRM University for providing the facilities to carry out the work. The authors are also thankful to Madras Pharmaceuticals, Chennai for providing the standard drugs as gift samples.

\section{Conflicts of interest}

Authors declare that there is no conflict of interest.

\section{References}

1. Lakshmi S, Lakshmi KS. H-Point Standard Addition Method for Simultaneous Spectrophotometric Determination of Irbesartan, hydrochlorothiazide and Telmisartan in tablets. IJPRC. 2014;4(2):373-380.

2. Srinivasa RK, Minakshi P, Nargesh KK. Spectrophotometric methods for the simultaneous estimation of losartan potassium and hydrochlorothiazide in tablet dosage forms. Chronicles of young scientists. 2011;2(3):155-160.

3. Sunil S, Ajit K, Hemendra G. Simultaneous Estimation of valsartan and hydrochlorothiazide solid dosage form using UV Spectroscopy. Bulletin of Pharmaceutical Research. 2011;1(3):10-12.

4. Rahman N, Ahmad Y, Azmi SNH. Kinetic Spectrophotometric Determination of Ramipril in Pharmaceutical Formulations. AAPS PharmSciTech. 2005;6(3):543-551.

5. Chaitali T, Jyoti D, Pawr PY. Simultaneous estimation and validation of losartan potassium and hydrochlorothiazide in bulk and tablet dosage form using different spectrophotometric method. Der Pharma Chemica. 2004;6(2):24-30.

6. El-Gindy A, Ashour A, Abdel FL, et al. Spectrophotometric determination of benazepril hydrochloride and hydrochlorothiazide in binary mixture using second derivative, second derivative of the ratio spectra and chemometric methods. J Pharm Biomed Anal. 2001;25(2):299-307. 
7. Attia MS. Spectrofluorimetric Assessment of Ramipril using Optical Sensor Samarium Ion-Doxycycline Complex Doped in Sol-Gel Matrix. J Pharm Biomed Anal. 2001;51(1):7-11.

8. Abdellatef HE. Spectrophotometric and Spectrofluorimetric Methods for the Determination of Ramipril in its Pure and Dosage Form. Spectrochim. Spectrochim Acta A Mol Biomol Spectrosc. 2007;66(3):701-706.

9. Naguib IA, Abdelaleem EA, Draz ME, et al. Linear Support Vector regression and partial least squares chemometric models for determination of hydrochlorothiazide and Benezapril hydrochloride in presence of related impurities: a comparative study. Spectro Chim Acta A Mol Biomol Spectrosc. 2014;130:350-356.

10. Darwish HW, Hassan SA, Salem MY, et al. Comparitive study between derivative spectrophotometry and multivariate calibration as analytical tools applied for the simultaneous quantitation of amlodipine, valsartan and Hydrochlorothiazide. Spectro Chim Acta A Mol Biomol.Spectrosc. 2013;113:215-223

11. Lakshmi KS, Lakshmi S. Simultaneous spectrophotometric determination of valsartan and hydrochlorothiazide by H-point standard addition method and partial least square regression. Acta Pharm. 2011;61(1):37-50.

12. Hegazy MA, Metwaly FH, Abdelkawy M, et al. Spectrophotometric and chemometric determination of hydrochlorothiazide and spironolactone in binary mixture in the presence of their impurities and degradants. Drug Test Anal. 2010;2(5):243-251.

13. Kaya B, Erdal D, Dumitru B. Chemometric methods for the simultaneous spectrophotometric determination of telmisartan and hydrochlorothiazide in the commercial pharmaceuticals. Rev Chem. 2009;60(6):544-550.

14. Dinc E, Ustundag O. Spectrophotometric quantitative resolution of hydrochlorothiazide and spironolactone in tablets by chemometric analysis methods. Farmaco. 2003;58(11):1151-1161.

15. Alanazi AM, Abdelhameed AS, Khalil NY, et al. HPLC method with monolithic column for simultaneous determination of irbesartan and hydrochlorothiazide in tablets. Acta Pharm. 2014;64(2):187-198.

16. Koyuturk S, Can NO, Atkosar Z, et al. A novel dilute and shoot HPLC assay method for quantification of irbesartan and hydrochlorothiazide in combination tablets and urine using second generation $\mathrm{C} 18$ bonded monolithic silica column with double gradient elution. J Pharm Biomed Anal. 2014;97:103-110.

17. Goswami N. A validated stability indicating liquid chromatographic method for determination of process related impurities and degradation behaviour of irbesartan in solid dosage form. $J$ Adv Pharm Tech Res. 2014;5(1):33-40.

18. Vujić Z, Mulavdić N, Smajić M, et al. Simultaneous analysis of irbesartan and hydrochlorothiazide: an improved HPLC method with the aid of the chemometric protocol. Molecules. 2012;17(3):3461-3474.

19. Rawool ND, Venkatachalam A. Analytical method for the simultaneous estimation of hydrochlorothiazide and metoprolol tartrate using RP HPLC. Indian J Pharm Sci. 2011;73(2):219-223.

20. Anand BK, Vinoth KG, Lakshmi Sivasubramanian. Simultaneous estimation of Ramipril and Amlodipine in pharmaceutical dosage form by RP-HPLC method. International Journal of Pharmacy and Pharmaceutical Sciences. 2011;3(4):196-198.

21. Bae SK, Kim MJ, Shim EJ, et al. HPLC Determination of Irbesartan in Human Plasma: Its Application to Pharmacokinetic Studies. Biomed Chromatogr. 2009;23(6):568-572.

22. Ferreirós N, Iriarte G, Alonso RM, et al. Separation and Quantitation of Several Angiotensin II Receptor Antagonist Drugs in Human Urine by a SPE-HPLC-DAD Method. J Sep Sci. 2008;31(4):667-676.

23. Najma S, Saeed AM, Shahid AS, et al. Simultaneous Determination of Olmesartan Medoxomil and Irbesartan and Hydrochlorothiazide in Pharmaceutical Formulations and Human Serum using High Performance Liquid Chromatography. Se Pu. 2008;26(5):544-549.

24. Ivanovic D, Medenica M, Jancic B, et al. Validation of analytical procedure for simultaneous determination of hydrochlorothiazide and lisinopril and their impurities. Acta chromatographica. 2007; 18:143-156.

25. Ferreirós N, Iriarte G, Alonso RM, et al. Development of a Solid Phase Extraction Procedure for HPLC-DAD Determination of Several Angiotensin II Receptor Antagonists in Human Urine using Mixture Design. Talanta. 2007;73(4):748-756.

26. Shakya AK, Al-Hiari YM, Alhamamib OMO. Liquid Chromatographic Determination of Irbesartan in Human Plasma. J Chromatogr B Analyt Technol Biomed Life Sci. 2007;848(2):245-250.

27. Patel LJ, Suhagia BN, Shah PB, et al. Simultaneous estimation of bisoprolol fumarate and hydrochlorothiazide in tablet dosage form by RP-HPLC method. Indian Journal of Pharmaceutical Sciences. 2006;8(5):635-638.

28. Caudron E, Laurent S, Billaud EM, et al. Simultaneous Determination of the Acid/Base Antihypertensive Drugs Celiprolol, Bisoprolol and Irbesartan in Human Plasma by Liquid Chromatography. J Chromatogr B Analyt Technol Biomed Life Sci. 2004;801(2):339-345.

29. Erk N. Simultaneous Determination of Irbesartan and Hydrochlorothiazide in Human Plasma by Liquid Chromatography. $J$ Chromatogr B Analyt Technol Biomed Life Sci. 2003;784(1):195-201.

30. Nie J, Zhang M, Fan Y, et al. Biocompatible In-Tube Solid Phase Microextraction Coupled to HPLC for the Determination of Angiotensin II Receptor Antagonists in Human Plasma and Urine. J Chromatogr B Analyt Technol Biomed Life Sci. 2003;828(1-2):62-69.

31. González L, López JA, Alonso RM, et al. Fast Screening Method for the Determination of Angiotensin II Receptor Antagonists in Human Plasma by High-Performance Liquid Chromatography with Fluorimetric Detection. J Chromatogr A. 2002;949(1-2):49-60.

32. Hertzog DL, McCafferty JF, Fang X, et al. Development and validation of a stability-indicating HPLC method for the simultaneous determination of Losartan potassium, hydrochlorothiazide, and their degradation products. J Pharm Biomed Anal. 2002;30(3):747-760.

33. Erk N. Analysis of binary mixtures of losartan potassium and hydrochlorothiazide by using high performance liquid chromatography, ratio derivative spectrophotometric and compensation technique. $J$ Pharm Biomed Anal. 2001;24(4):603-611.

34. Belal F, Al-Zaagi IA, Gadkariem EA, et al. A Stability Indicating LC Method for the Determination of Ramipril and Hydrochlorothiazide in Dosage Forms. J Pharm Biomed Anal. 2001;24(3):335-342.

35. Hogan BL, Williams M, Idiculla A, et al. Development and Validation of a Liquid Chromatographic Method for the Determination of the Related Substances of Ramipril in Atlace Capsules. J Pharm Biomed Anal. 2000;23(4):637-651.

36. Qui X, Wang Z, Wang B, et al. Simultaneous determination of irbesartan and hydrochlorothiazide in human plasma by ultra high performance liquid chromatography tandem mass spectrometry and its application to the bioequivalence study. J Chromatogr B Analyt Technol Biomed Life Sci. 2014;957:110-115.

37. Chi YL, Chia HF. Quantitation of Irbesartan and Major Proteins in Human Plasma by Mass Spectrometry with Time-of-Flight Analyzer. $J$ Pharm Biomed Anal. 2011;54(1):100-105.

38. Prasaja B, Sasongko L, Harahap Y, et al. Simultaneous quantification of Losartan and active metabolite in human plasma by LC-MS using irbesartan as internal standard. $J$ Pharm Biomed Anal. 2009;49(3):862-867. 
39. Lee HW, Ji HY, Park ES, et al. Hydrophilic Interaction ChromatographyTandem Mass Spectrometric Analysis of Irbesartan in Human Plasma: Application to Pharmacokinetic Study of Irbesartan. J Sep Sci. 2009;32(14):2353-2358.

40. Sathe SR, Bari SB. Simultaneous analysis of losartan potassium, atenolol, and hydrochlorothiazide in bulk and in tablets by high-performance thin-layer chromatography with UV absorption densitometry. Acta Chromatographica. 2007;19:270-278.

41. Kristoffersen L, Øiestad E, Opdal MS, et al. Simultaneous Determination of 6 Beta-Blockers, 3 Calcium Channel Antagonists, 4 Angiotensin-II Antagonists and 1 Antiarrhythmic Drug in Post-Mortem Whole Blood by Automated Solid Phase Extraction and Liquid Chromatography Mass Spectrometry. Method Development and Robustness Testing by Experimental Design. J Chromatogr B Analyt Technol Biomed Life Sci. 2007;850(1-2):147-160.

42. Zhang M, Wei F, Zhang YF, et al. Novel Polymer Monolith Micro extraction using a Poly (Methacrylic Acid-Ethylene Glycol Dimethacrylate) Monolith and its Application to Simultaneous Analysis of Several Angiotensin- II Receptor Antagonists in Human Urine by Capillary Zone Electrophoresis. J Chromatogr A. 2006;1102(12):294-301.
43. Sharma M, Kothari C, Sherikar O, et al. Concurrent estimation of amlodipine besylate, hydrochlorothiazide and valsartan by RPHPLC, HPTLC and UV Spectrophotometry. J Chromatogr Sci. 2014;52(1):27-35.

44. Lakshmi KS, Lakshmi S. Simultaneous Analysis of Losartan Potassium, Amlodipine Besylate and Hydrochlorothiazide in bulk and in tablets by HPTLC with UV Absorption Densitometry. J Anal Methods Chem. 2012;2012:108281.

45. Kumbhar ST, Chougule GK, Tegeli VS, et al. A validated HPTLC method for simultaneous quantification of nebivolol and hydrochlorothiazide in bulk and tablet formulation. International Journal of Pharmaceutical Sciences and Drug Research. 2011;3(1):62-66.

46. Panchal HJ, Suhagia BN. Simultaneous determination of Atorvastatin calcium and ramipril in capsule dosage forms by HPLC and HPTLC. $J$ AOAC Int. 2010;93(5):1450-1457.

47. Lakshmi KS, Lakshmi S, Krishanu Pal. Stability indicating HPTLC method for simultaneous determination of Ramipril and Telmisartan in tablets. International Journal of Pharmacy and Pharmaceutical Sciences. 2010;2(4):127-129. 